\title{
Endoscopic ultrasound (EUS)-guided ethanol injec- tion in hepatic metastatic carcinoma: a case report
}

Endoscopic ultrasound (EUS)-guided ethanol injection is used as palliative treatment for localized pancreatic tumorous lesions such as pancreatic cysts [1]. However, there have been few reports of its use in hepatic metastatic carcinoma. Here we report the use of EUS-guided ethanol injection for treatment of hepatic metastatic carcinoma following pancreatoduodenectomy in a patient with pancreatic cancer, which was successful in obtaining local control.

A 47-year-old Chinese man presented to Changhai Hospital with a 3-month history of abdominal pain and jaundice. A computed tomographic (CT) scan revealed pancreatic cancer with enlargement of the intrahepatic and extrahepatic bile ducts. The serum level of CA19-9 was 268.5 U/L (normal range $0-37 \mathrm{U} / \mathrm{L}$ ) and cytology confirmed malignant cells on EUS-guided fine-needle aspiration (FNA). Following pancreatoduodenectomy, histopathological examination confirmed the presence of acinar cell carcinoma in the head of pancreas. The patient underwent seven courses of chemotherapy with gemcitabine $1.2 \mathrm{~g}$ with an interval of 7 days between the courses. After 17 months of the operation, the patient reported upper abdominal pain. An enhanced abdominal CT scan showed recurrence of pancreatic cancer and metastases in the retroperitoneal lymph nodes. Cytology of lymph nodes confirmed the recurrence of pancreatic cancer. EUS-guided radioactive iodine125 seed implantation was carried out; the radioactive seeds were implanted into the enlarged lymph nodes and the pancreatic cancer mass, using a $19 \mathrm{~T}$ needle ( $\bullet$ Fig. 1), a method that we have reported previously [2].

After the operation, there was no abdominal pain and the patient received eight courses of chemotherapy using the same protocol as before. However, a CT scan after the last course of chemotherapy revealed hepatic porta and left hepatic metastatic carcinoma ( Fig. 2 ), which was confirmed by EUS-FNA.

We decided to use ethanol injection to treat the hepatic metastatic carcinoma. Because a part of the lesion was located

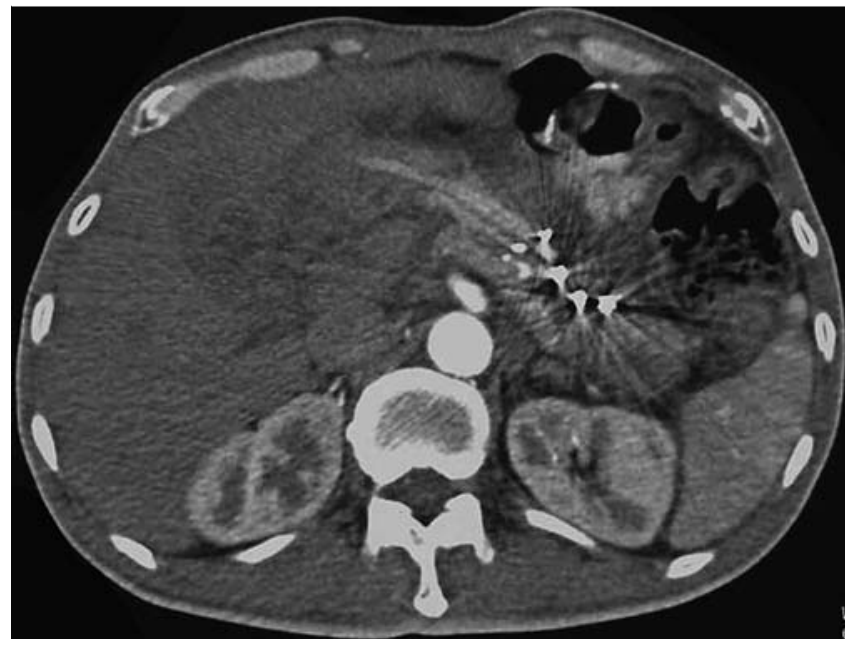

Fig. 1 Computed tomographic (CT) scan after implantation of radioactive iodine- 125 seeds.

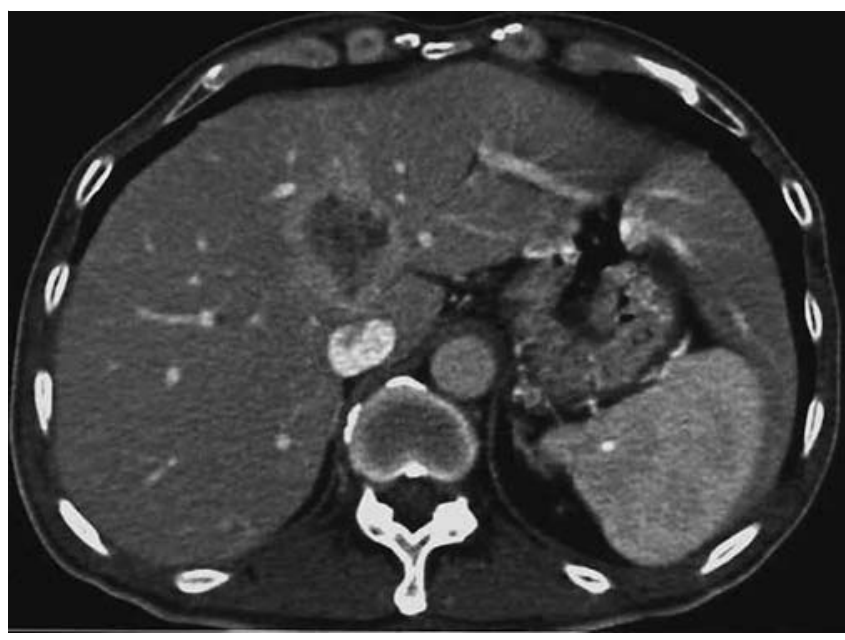

Fig. 2 Computed tomographic (CT) scan showed the hepatic porta and hepatic metastatic carcinoma on the left side.

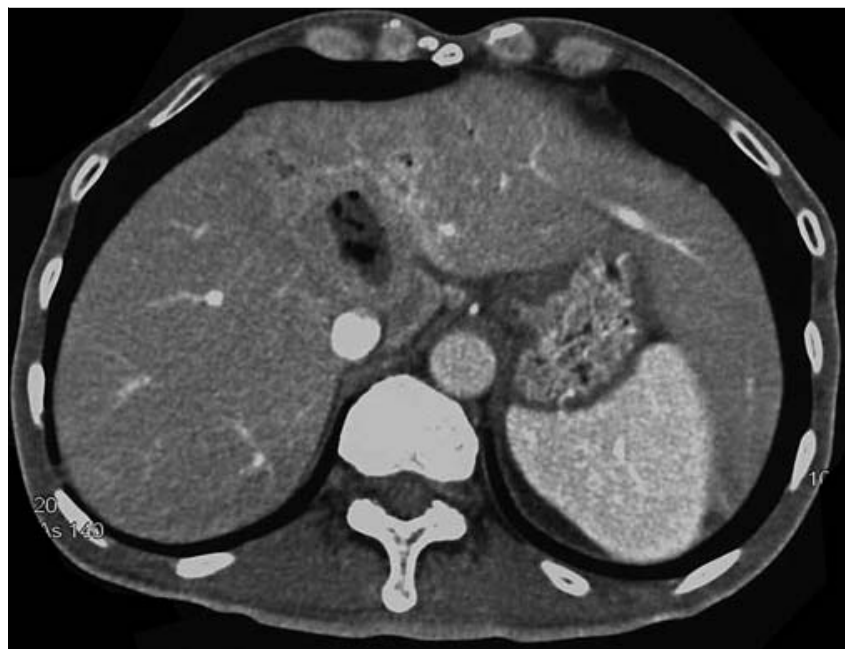

Fig. 3 Computed tomographic (CT) scan showed liquefaction and reduction in size of the hepatic metastatic carcinoma after ethanol injection. 
within the hepatic porta, we could not fully avoid injuring important blood vessels and the bile duct. In view of the advantages of EUS-FNA in diagnosis and treatment of the hepatic disease, we chose to carry out EUS-guided ethanol injection therapy. The procedure was successful, and there were no significant procedure-related complications. The injection of ethanol was safe for the patient, expect for low-grade fever. Laboratory tests revealed a normal hemogram and no significant changes in liver function. At 1 month after the ethanol injection, we found most of the metastatic carcinoma in the liver had liquefied; thus we had achieved local control with decrease in size of the lesion as compared with the previous abdominal CT scan ( $\bullet$ Fig. 3 ). We are unaware of any previous reports similar to this case report. On the basis of the present case, we conclude that use of ethanol injection for metastatic carcino- ma should be safe and effective, and that EUS-guided ethanol injection treatment for hepatic metastatic carcinoma will be a topic for research in the future.

\section{Competing interests: None}

Endoscopy_UCTN_Code_TTT_1AS_2AG

Y.-H. Hu ${ }^{1 *}$, X.-P. Tuo ${ }^{1 *}$, Z.-D. Jin ${ }^{2}$, Y. Liư $^{2}$, Y. Guo ${ }^{2}$, L. Luo ${ }^{1}$

1 Department of Geriatrics, Changhai Hospital, Shanghai, China

2 Gastroenterology Division, Digestive Endoscopy Center of Changhai Hospital, Shanghai, China

${ }^{*}$ Y.-H. Hu and X.-P. Tuo are co-first authors.

\section{References}

1 Gan SI, Thompson CC, Lauwers GY et al. Ethanol lavage of pancreatic cystic lesions: initial pilot study. Gastrointest Endosc 2005; 61: $746-752$

2 Jin Z, Du Y, Li Z et al. Endoscopic ultrasonography-guided interstitial implantation of iodine 125-seeds combined with chemotherapy in the treatment of unresectable pancreatic carcinoma: a prospective pilot study. Endoscopy 2008; 40: 314-320

\section{Bibliography}

DOI $10.1055 / \mathrm{s}-0030-1255653$

Endoscopy 2010; 42: E256 -E257

(c) Georg Thieme Verlag KG Stuttgart · New York . ISSN 0013-726X

\section{Corresponding author}

Z.-D. Jin

Digestive Endoscopy Center of Changhai Hospital, Gastroenterology

The Second Military Medical University

168 Changhai Road

Shanghai 200433

China

zhendjin@126.com 